\title{
Comparative Evaluation of Resin-modified Glass Ionomer Cement, Mineral Trioxide Aggregate, and Calcium Hydroxide When Used as a Direct Pulp Capping Material on Carious Pulp Exposures of Human Permanent Teeth: A Randomized Clinical Trial
}

\author{
M Agrawal Aanchal ${ }^{1}$, Vanitha U Shenoy ${ }^{2}$, V Margasahayam Sumanthini ${ }^{3}$, S Satpute Tanvi ${ }^{4}$
}

\begin{abstract}
Aim and objective: To evaluate the clinical efficacy of resin-modified glass ionomer cement (RMGIC), mineral trioxide aggregate (MTA), and calcium hydroxide $(\mathrm{CH})$ when used as a direct pulp capping (DPC) material on carious exposure of human permanent teeth.

Materials and methods: Forty-five participants in the age group of 15-52 years, with deep carious lesions diagnosed with reversible pulpitis, were selected and divided into three groups, i.e., group I: RMGIC, group II: MTA, and group III: CH. Recall evaluation after 24 hours, 3 weeks, 3 months, and 6 months was carried out for recording self-reports from participants, a clinical inspection of the tooth, evaluating radiographs, testing vitality, palpation, percussion, mobility, and probing depths.

Results: Of the 45 participants, 42 presented without any abnormal signs or symptoms. Three participants presented with symptoms of irreversible pulpitis, which indicated DPC failure. Chi-square test gave a $p$ value $>0.05$.

Conclusion: There was no significant association between different parameters and the DPC materials used in the study.

Clinical significance: Carious pulp exposure poses a challenge to the endodontist and maintaining the vitality of the pulp by DPC is one of the treatment modalities. This study has attempted to compare the efficacy of commonly used pulp capping materials like MTA and CH in addition to RMGIC, which has routinely been used for restorations.

Keywords: Calcium hydroxide, Direct pulp capping, Mineral trioxide aggregate, Resin-modified glass ionomer cement.

World Journal of Dentistry (2021): 10.5005/jp-journals-10015-1862
\end{abstract}

\section{INTRODUCTION}

Preservation and maintenance of pulp tissue in a healthy state after it has been affected by caries/the tooth underwent trauma or a restorative procedure is known as vital pulp therapy. ${ }^{1}$

In such cases when a biocompatible material is placed directly over the exposed pulp, it is referred to as a direct pulp capping (DPC) procedure, wherein the main aim will be to maintain the vitality and the health of the pulp. ${ }^{2}$ Various materials have been used as pulp capping (PC) materials over the years ${ }^{3}$ but calcium hydroxide (CH) has been one such material ${ }^{4}$ by which others were judged.

Calcium hydroxide, as a VPT material, has shown acceptable properties but variable outcomes have been observed in longterm studies 5 as $\mathrm{CH}$ has demonstrated cytotoxicity in cell cultures, its inability to stimulate reparative dentin coupled with poor marginal adaptation to dentin and inducing of pulp cell apoptosis. ${ }^{6}$ Favorable pulp responses following the use of mineral trioxide aggregate (MTA $)^{7}$ which is a bioactive silicate cement that is an effective PC material in canine models and non-human primates. ${ }^{8,9}$ Resin-modified glass ionomer cement (RMGIC) has been used for DPC. Resin-modified glass ionomer cements have been reported to be cytotoxic to the pulp ${ }^{10}$ found to have a favorable response in a deep cavity as a liner. ${ }^{11}$ Yet information is scarce about the effects of RMGIC as a DPC material. ${ }^{12}$ To date, few clinical studies have investigated the effect of RMGIC as a DPC material. ${ }^{13-15}$ Thus, this study aims to evaluate the clinical efficacy of RMGIC, MTA, and
${ }^{1-4}$ Department of Conservative Dentistry and Endodontics, MGM Dental College and Hospital, Kamothe, Navi Mumbai, Maharashtra, India

Corresponding Author: Vanitha U Shenoy, Department of Conservative Dentistry and Endodontics, MGM Dental College and Hospital, Kamothe, Navi Mumbai, Maharashtra, India, Phone: +91 9167629730, e-mail: vanithashenoy@gmail.com

How to cite this article: Aanchal MA, Shenoy VU, Sumanthini VM, et al. Comparative Evaluation of Resin-modified Glass lonomer Cement, Mineral Trioxide Aggregate, and Calcium Hydroxide When Used as a Direct Pulp Capping Material on Carious Pulp Exposures of Human Permanent Teeth: A Randomized Clinical Trial. World J Dent 2021;12(5):381-385.

Source of support: Nil

Conflict of interest: None

$\mathrm{CH}$ when used as a DPC material in carious exposures of human permanent teeth.

\section{Materials and Methods}

Subjects were recruited between June 2015 and June 2016 for this clinical study. Approval for the project was obtained from the Institutional Ethics Committee for Research on Human 
Subjects (MGM/DCH/IEC/30/12/2014). The participants were explained the procedure of DPC which included the rationale, the clinical procedure that would be undertaken, and the possible complications of DPC and then informed consent was obtained. The participants were treated in accordance with the Helsinki Declaration. The inclusion criteria being: participants in the age group of 15-52 years, permanent maxillary and mandibular 1st and 2 nd molars exhibiting carious pulp exposure. An intraoral periapical radiograph (Fig. 1A) of the tooth in concern was taken, tooth mobility checked, periodontal probing depth recorded, tooth percussed for periapical status, and vitality of the tooth was assessed using cold spray and an electric pulp tester. Those teeth which were diagnosed as having reversible pulpitis were included.

The exclusion criteria were participants with teeth diagnosed as having irreversible pulpitis from their history or clinical examination revealing the presence of a sinus tract or periodontal inflammation, mobility of tooth with poor prognosis, cracked tooth, furcation involvement, resorptive defects, or calcification of the root canal. During the clinical procedure of the participant included in the study, if it was observed that the pulpal hemorrhage could not be controlled and the same lasted for $>10$ minutes, such teeth were excluded from the study. The medical history of all participants was non-contributory.

A sample size of 45 participants was arrived at (15 participants per group were selected), which set the power of the study at $90 \%$ and precision for the study was $9 \%$. All participants were assigned into the three groups with the help of simple random sampling using sealed envelopes. The clinical procedure was carried out by the primary investigator and the participant allocation was done by a neutral investigator, who did not carry out any clinical procedure. The participants did not know which PC material was being placed and the statistician also was unaware of the materials used per group. The primary investigator was aware of the material being placed.

All DPC procedures were carried out under local anesthesia 2\% (LOX 2\%, NEON, India) and rubber dam isolation (Hygienic, India) (Figs $1 \mathrm{~A}$ and $\mathrm{C}$ ).

A sterile carbide bur \#245, in a high-speed handpiece, with water coolant was used to remove the undermined and carious enamel.
Large round stainless steel bur in a slow-speed handpiece was used for the excavation of caries. Caries on the pulpal or axial walls were excavated using a spoon or discoid excavators (Fig. 1D), the removal of which resulted in the exposure of the pulp (Fig. 1E), which was $\leq 0.5 \mathrm{~mm}$ in diameter. Once the exposure was detected, the cavity was disinfected by irrigating with $3 \%$ sodium hypochlorite (NaOCl) (Prime Dental Products Pvt Ltd, India) to remove all the debris and the bleeding from the pulp exposure site was controlled by placing a $3 \% \mathrm{NaOCl}$ moistened sterile cotton pellet, applying moderate pressure to the exposed pulp for a maximum of 10 minutes to achieve hemostasis. Once the bleeding was controlled, the cavity was irrigated slowly with $1 \mathrm{~mL}$ of normal saline (Baxter India Pvt. Ltd., India) to remove any remnants of $\mathrm{NaOCl}$. Finally, the cavity was lightly dabbed with saline moistened sterile cotton pellet to remove excess moisture. In case of uncontrollable bleeding, i.e., $>10$ minutes, irreversible pulpal damage was assumed and the participant was excluded from the study.

The PC material was then applied according to the manufacturer's instructions (Fig. 1F). The thickness of all three PC materials was a maximum of $1.5 \mathrm{~mm}$ over the pulp exposure leaving $1-2 \mathrm{~mm}$ of dentin and enamel available circumferentially for the future liner placement. The final restoration included placement of a polycarboxylate liner (Harvard Polycarboxylate Cement, Harvard Dental Intl, Germany) (Fig. 1G) and restoration of the cavity with silver amalgam (ARISTA-ALLOY 21, Cookson Precious Metals Ltd., Birmingham, UK) (Fig. 1H).

For group I: RMGIC (GC India Dental Pvt Ltd) was directly placed over the exposure site and the surrounding dentin using a plastic filling instrument with a small round tip then light-cured for 20 seconds using a visible light curing device (COXO, DB-685, Penguin, Europe) (470 $\mathrm{nm}$ wavelength, intensity $1,200 \mathrm{~mW} / \mathrm{cm}^{2}$ ). The light source was placed as close as possible to the cement surface.

For group II: MTA: following manipulation, MTA was placed over the pulp exposure and also the dentin surrounding the exposure, followed by placing a sterile cotton pellet moistened with sterile distilled water, over the MTA, and the tooth was provisionally restored with intermediary restorative material (IRM) (Dentsply, USA) for 24 hours, after which the temporary restoration and the cotton pellet was removed ${ }^{3}$ and final restoration done as described above.
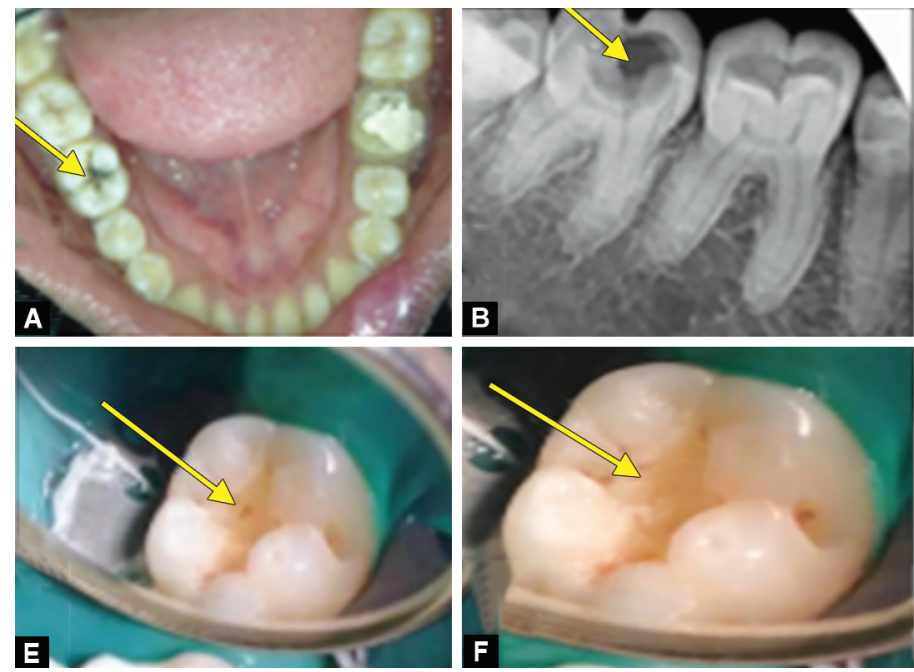
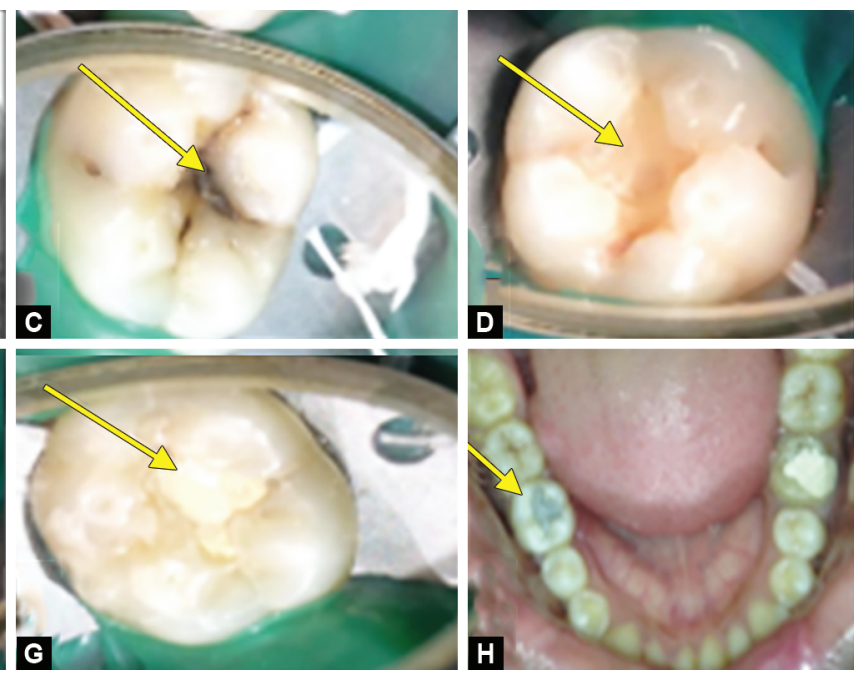

Figs 1 A to H: 36 (A) Preoperative photograph; (B) Preoperative radiograph; (C) Rubber dam isolation; (D) After caries excavation; (E) Pinpoint pulp exposure noted; (F) Placement of RMGIC pulp capping agent; (G) Polycarboxylate base placed; (H) Silver amalgam restoration 
For group III: $\mathrm{CH}$ : following manipulation, $\mathrm{CH}$ (GC India Dental Pvt. Ltd.) cement was placed over the pulp exposure and also the dentin surrounding the exposure. After the complete setting of the $\mathrm{CH}$, the final restoration was done as described above.

Recall evaluation of the following parameters for each participant was at 24 hours, 3 weeks, 3 months, and 6 months:

- Recording self-reports from participants (S)

- Clinical inspection of the tooth (I)

- Evaluating radiographs for normal and pathological changes (R)

- Testing the pulp-capped teeth for vitality by electric and cold tests (V)

- Palpation (PA)

- Percussion (PE)

- Mobility (M)

- Probing depths (PD).

Statistical analysis was done using SPSS software version 16 . The Chi-square test was applied to check for an association between different parameters and the groups. In addition, a 95\% confidence interval for the success rate for all the 3 groups was calculated. A $p$ value $<0.05$ was considered statistically significant.

\section{Results}

All 45 participants returned for follow-up, 42 were asymptomatic and 03 of them presented with continuous pain, not relieved by medication, which were suggestive of irreversible pulpitis, and were excluded from the study. A consort flow diagram showing the enrollment, allocation, follow-up of all the participants included in this clinical study is as presented in Flowchart 1.

Group-wise comparison of signs and symptoms at the 6-month follow-up period (Table 1) showed that 15 participants from the RMGIC group, 13 participants from the MTA group, and 14 participants from the $\mathrm{CH}$ group did not complain of pain (Fig. 2), vitality testing revealed normal findings (Fig. 3). Vertical percussion elicited a negative response. Group-wise comparison of signs and symptoms at 24 hours, 3 weeks, 3 months, and 6 months are presented in Figures 2 to 4 . Between the 3rd and 6th month follow-up, two participants from the MTA group and one participant from the $\mathrm{CH}$ group showed all signs and symptoms resembling irreversible pulpitis, and a decision was made to initiate endodontic treatment for these three participants (Figs 2 to 4 ). Since the $p$ value for the Chi-square is $>0.05$, indicates no significant association between different parameters and the groups.

\section{Discussion}

VPT aims to maintain the coronal and radicular pulp tissue in a viable condition by preserving its vitality. ${ }^{1}$ Direct pulp capping is one such procedure in which an exposed dental pulp is covered with a suitable material that protects the pulp from additional injury and permits healing and repair ${ }^{16}$ and alternative to root canal therapy or extraction. In group I, RMGIC was the PC material used. Conventional GIC and RMGICs have been used a PC agents since the early $90 \mathrm{~s} .{ }^{13,14,17}$

Resin-modified glass ionomer cement combines the main advantages of glass-ionomer cements such as adhesion to the tooth structure, fluoride release, and biocompatibility, with the easy handling of light polymerization. ${ }^{18}$ In the RMGIC group, it was observed that none of the participants had any complaint with respect to the pulp-capped tooth at the end of the study period of 6 months. Shameem et al. used Vitrebond ${ }^{T M}$, resin-modified glass ionomer liner and Vitremer ${ }^{\mathrm{TM}}$, resin-modified glass ionomer cement $(\mathrm{GIC})$ in comparison with the control group of Dycal ${ }^{\circledR}$ as PC agents in iatrogenic exposures and observed that reparative dentin formed is comparable to that of reparative dentin formed with

Flowchart 1: CONSORT flow diagram

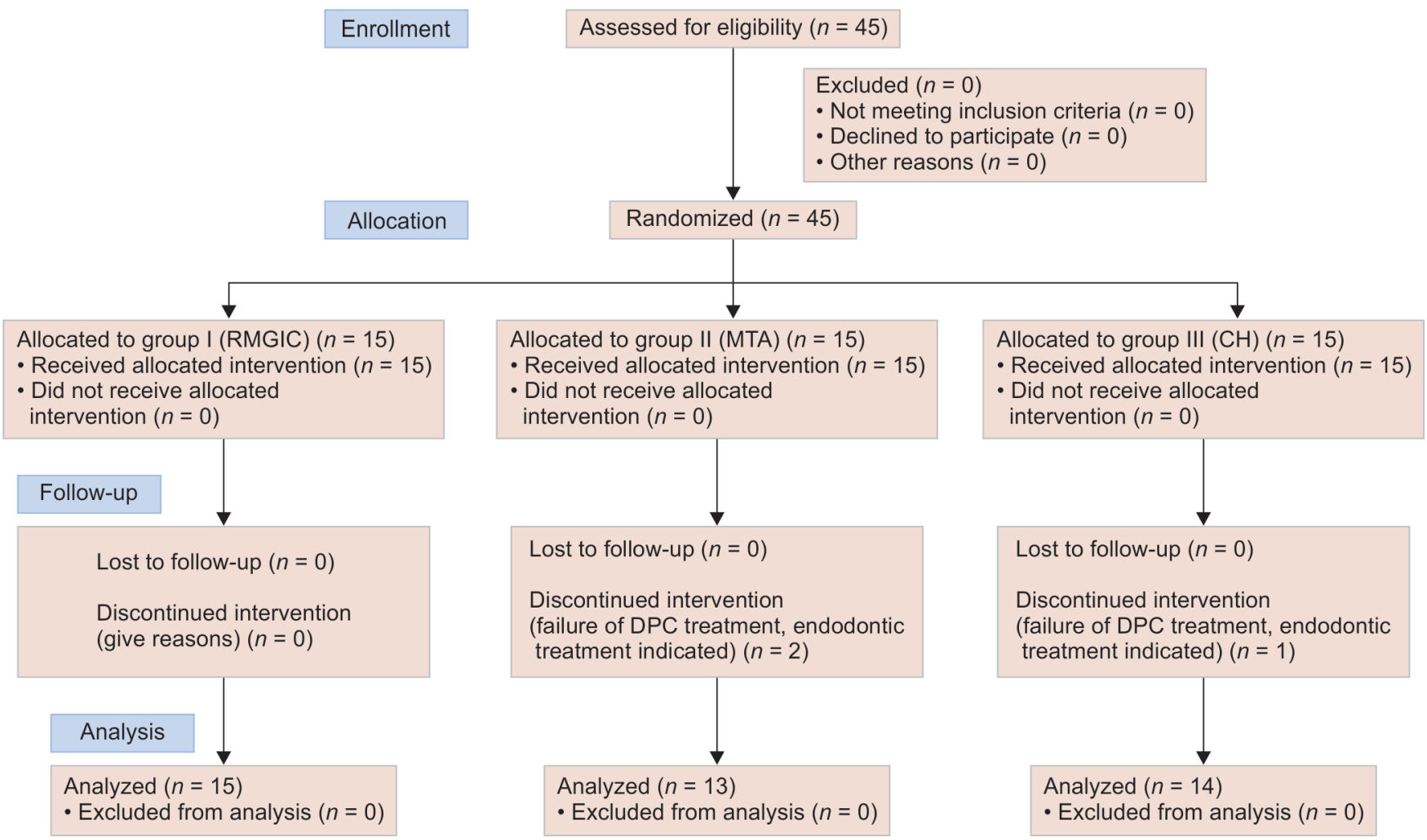


Table 1: Group-wise comparison of signs and symptoms at 6 months

\begin{tabular}{|c|c|c|c|c|c|c|c|c|}
\hline & & & \multicolumn{6}{|c|}{ Groups } \\
\hline & & & \multicolumn{2}{|c|}{ Rmgic } & \multicolumn{2}{|c|}{ MTA } & \multicolumn{2}{|c|}{ Calcium hydroxide } \\
\hline & & & Count & Column N\% & Count & Column N\% & Count & Column N\% \\
\hline \multirow[t]{2}{*}{1.} & $\mathrm{~S}$ & Present & 0 & 0 & 0 & 0 & 1 & 7.1 \\
\hline & & Absent & 15 & 100.0 & 13 & 100.0 & 13 & 92.9 \\
\hline \multirow[t]{2}{*}{2.} & I & Normal & 15 & 100.0 & 13 & 100.0 & 14 & 100.0 \\
\hline & & Abnormal & 0 & 0 & 0 & 0 & 0 & 0 \\
\hline \multirow[t]{2}{*}{3.} & V & Normal & 15 & 100.0 & 13 & 100.0 & 14 & 100.0 \\
\hline & & Abnormal & 0 & 0 & 0 & 0 & 0 & 0 \\
\hline \multirow[t]{2}{*}{4.} & $\mathrm{PA}$ & Positive & 0 & 0 & 0 & 0 & 0 & 0 \\
\hline & & Negative & 15 & 100.0 & 13 & 100.0 & 14 & 100.0 \\
\hline \multirow[t]{2}{*}{5.} & $\mathrm{PE}$ & Positive & 0 & 0 & 0 & 0 & 0 & 0 \\
\hline & & Negative & 15 & 100.0 & 13 & 100.0 & 14 & 100.0 \\
\hline \multirow[t]{2}{*}{6.} & $M$ & Present & 0 & 0 & 0 & 0 & 0 & 0 \\
\hline & & Absent & 15 & 100.0 & 13 & 100.0 & 14 & 100.0 \\
\hline \multirow[t]{2}{*}{7.} & PD & Normal & 15 & 100.0 & 13 & 100.0 & 14 & 100.0 \\
\hline & & Abnormal & 0 & 0 & 0 & 0 & 0 & 0 \\
\hline \multirow[t]{2}{*}{8.} & $\mathrm{R}$ & Satisfactory & 15 & 100.0 & 13 & 100.0 & 14 & 100.0 \\
\hline & & Unsatisfactory & 0 & 0 & 0 & 0 & 0 & 0 \\
\hline
\end{tabular}

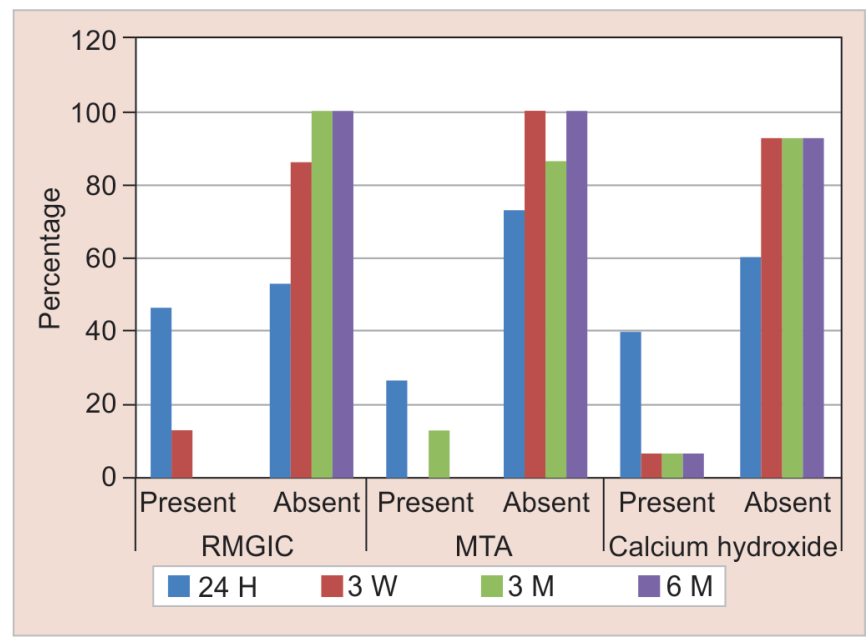

Fig. 2: Bar graph depicts presence or absence of pain

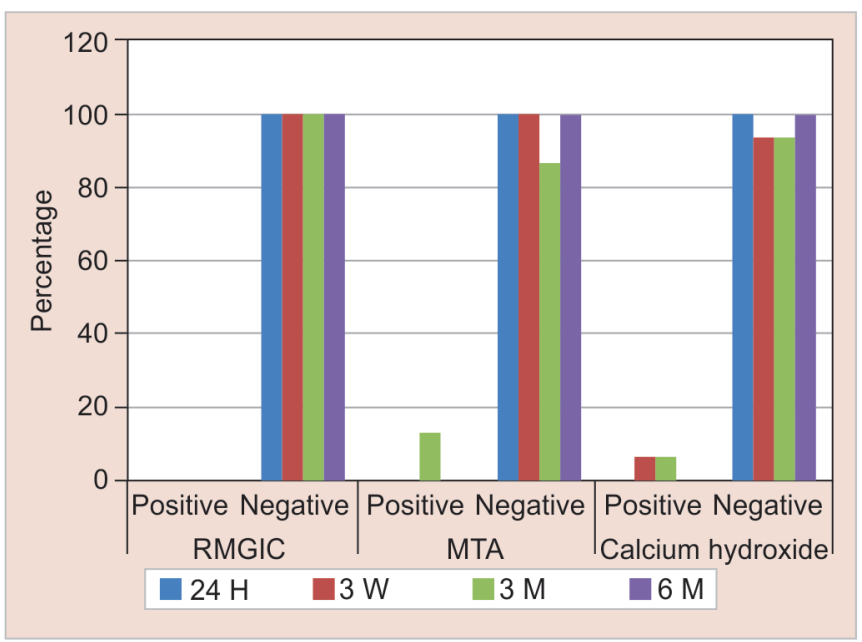

Fig. 4: Bar graph depicts presence/absence of tenderness to percussion

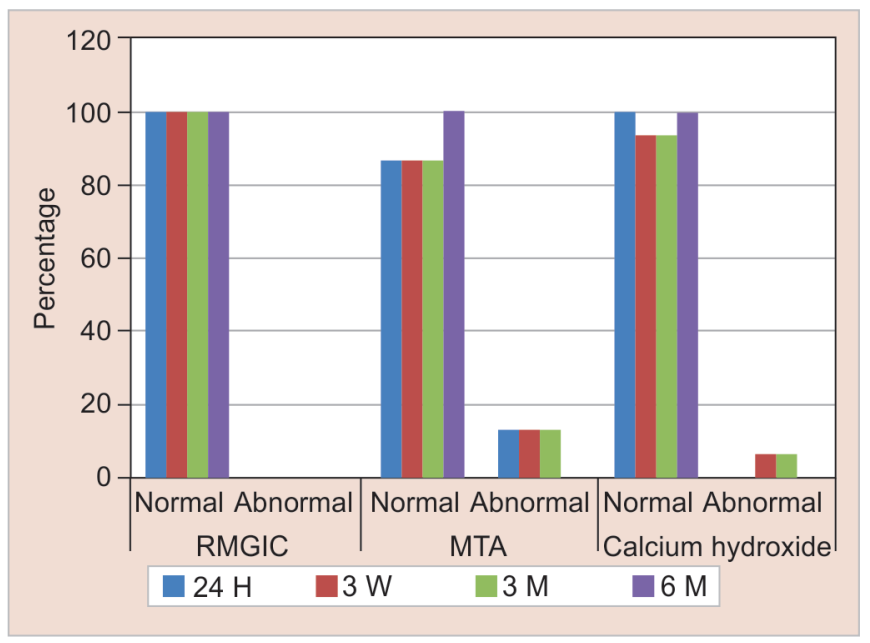

Fig. 3: Bar graph depicts vitality

time-proven $\mathrm{CH}^{19}{ }^{19}$ Resin-modified glass ionomer cements have been considered as less biocompatible due to the release of unreacted monomers and other components which has discouraged the materials being used to cap the exposed pulp in human participants. However, Tarim et al. ${ }^{12}$ in their animal study on exposed as well as non-exposed pulps in monkeys showed acceptable biological compatibility of the RMGIC for this application in both scenarios, confirming the results of a study done by Felton et al. ${ }^{13}$ using an experimental RMGIC. Vitremer/Vitrebond was the RMGICs used in all the above studies. The material used in the present study, i.e., Fuji II LC differed in its composition from the GICs used earlier like Vitremer/ Vitrebond, which is a tri-cured RMGIC containing potential leachable components including unreacted HEMA, camphorquinone (CQ), diphenyliodonium chloride (DC), tricarboxylic acid (TA), potassium persulfate $\left(\mathrm{K}_{2} \mathrm{~S}_{2} \mathrm{O}_{8}\right)$, and ascorbic acid responsible for the cytotoxicity. ${ }^{17}$ The photo-activator in Vitremer is DC but Fuji II LC uses $\mathrm{N}, \mathrm{N}$-dimethylaminoethyl methacrylate ${ }^{20}$ could be the reason the present study showed no failures. 
In group II, MTA was the PC material. Yasuda et al. ${ }^{21}$ compared MTA, Dycal, and adhesive resin cement. Their data indicated that MTA has no cytotoxicity after 72 hours and it not only significantly increases mineralization by stimulating dental pulp cells, but also increases the amount of bone morphogenetic protein-2 (BMP-2) production. Mineral trioxide aggregate as a DPC material has shown promising results ${ }^{3}$ and when compared with $\mathrm{CH}$, has advantageous properties such as bioactivity with antibacterial and antifungal effect $^{22}$ good biocompatibility, it is hydrophilic, radio-opaque, highly alkaline $\mathrm{pH}$ which makes it bacteriostatic, has the excellent sealing ability, low marginal leakage, and low solubility. ${ }^{8}$ When MTA and $\mathrm{CH}$ were used for DPC procedures for evaluating their effectiveness in maintaining long-term pulp vitality in clinical studies the authors observed that compared to $\mathrm{CH}$, MTA appeared to be more effective. ${ }^{2,7}$

In group III, CH was the PC material used. Considering the alkalinity, biocompatibility, and antimicrobial activity, it seems that $\mathrm{CH}$ is a suitable material for PC. However, its sealing ability has been observed to be not as complete as MTA, ${ }^{23}$ and solubility in fluids is a problem that requires a good coronal seal. It does not have any inherent adhesive quality. The other drawback of $\mathrm{CH}$ is the presence of tunnel defects in the reparative dentin has been observed when $\mathrm{CH}$ has been used as a DPC material. A tunnel defect is a passage from the exposure site through the reparative dentin to the pulp which may consist of fibroblasts and capillaries in the defect. ${ }^{24}$

The success of DPC depends on the ability of the operator to control bleeding after the pulp has been exposed during carious excavation and also before placing the $\mathrm{PC}$ material can affect the success of DPC. In the present study, a cotton pellet moistened with $3 \% \mathrm{NaOCl}$ was used to control the bleeding as it has antibacterial property and controls the hemorrhage efficiently. ${ }^{25}$ The reason for the failure of the three DPCs could be an inability to control bleeding or the size of the pulpal exposure may have exceeded $0.5 \mathrm{~mm}$.

The limitations of this study were that the sample size was small with a shorter follow-up period and the dentin bridge formation was not evaluated.

\section{CONCLUSION}

All the three materials-RMGIC, $\mathrm{CH}$, and MTA when used and evaluated as a DPC material over carious exposure of human permanent teeth provided a favorable result. There was no significant relationship between different parameters and the PC materials used in the study. Resin-modified glass ionomer cement can be recommended for DPC; however, further clinical studies need to be carried out.

\section{References}

1. Bogen G, Chandler NP. Vital pulp therapy. In: Ingle J, Bakland L, Baumgartner J, ed. Endodontics. 6th ed., Hamilton, Ontario: BC Decker;; 2008. pp. 1310-1313.

2. Mente J, Geletneky B, Ohle M, et al. Mineral trioxide aggregate or calcium hydroxide direct pulp capping: an analysis of the clinical treatment outcome. J Endod 2010;36(5):806-813. DOI: 10.1016/j. joen.2010.02.024.

3. Bogen G, Kim JS, Bakland LK. Direct pulp capping with mineral trioxide aggregate: an observational study. J Am Dent Assoc 2008;139(3):305-315. DOI: 10.14219/jada.archive.2008.0160quiz 305-15 Erratum in, J Am Dent Assoc 2008;139(5):541.

4. Hilton TJ. Keys to clinical success with pulp capping: a review of the literature. Oper Dent 2009;34(5):615-625. DOI: 10.2341/09-132-0.
5. Barthel CR, Rosenkranz B, Leuenberg A, et al. Pulp capping of carious exposures treatment outcome after 5 and 10 years: a retrospective study. J Endod 2000;26(9):525-528. DOI: 10.1097/00004770200009000-00010.

6. Schröder U. Effects of calcium hydroxide-containing pulp-capping agents on pulp cell migration, proliferation, and differentiation. J Dent Res 1985;64(Spec No):541-548. DOI: 10.1177/002203458506400407.

7. Hilton TJ, Ferracane JL, Mancl L. Northwest practice-based research collaborative in evidence-based dentistry (NWP). Comparison of $\mathrm{CaOH}$ with MTA for direct pulp capping: a PBRN randomized clinical trial. J Dent Res 2013;92(7 Suppl):16S-22S. DOI: 10.1177/0022034513484336.

8. Camilleri J, Pitt, Ford TR. Mineral trioxide aggregate: a review of the constituents and biological properties of the material. Int Endod J 2006;39(10):747-754. DOI: 10.1111/j.1365-2591.2006.01135.x.

9. Ford TR, Torabinejad $M$, Abedi HR, et al. Using mineral trioxide aggregate as a pulp-capping material. J Am Dent Assoc 1996;127(10):1491-1494. DOI: 10.14219/jada.archive.1996.0058.

10. Modena KC, Casas-Apayco LC, Atta MT, et al. Cytotoxicity and biocompatibility of direct and indirect pulp capping materials. J Appl Oral Sci 2009;17(6):544-554. DOI: 10.1590/s1678-77572009000600002.

11. Hse KM, Leung SK, Wei SH. Resin-ionomer restorative materials for children: a review. Aust Dent J 1999;44(1):1-11. DOI: 10.1111/j.18347819.1999.tb00529.x.

12. Tarim B, Hafez AA, Cox CF. Pulpal response to a resin-modified glass-ionomer material on nonexposed and exposed monkey pulps. Quintessence Int 1998;29(8):535-542.

13. Felton DA, Cox CF, Odom M, et al. Pulpal response to chemically cured and experimental light-cured glass ionomer cavity liners. J Prosthet Dent 1991;65(5):704-712. DOI: 10.1016/0022-3913(91)90210-n.

14. Murray PE, García-Godoy F. The incidence of pulp healing defects with direct capping materials. Am J Dent 2006;19(3):171-177.

15. do Nascimento AB, Fontana UF, Teixeira HM, et al. Biocompatibility of a resin-modified glass-ionomer cement applied as pulp capping in human teeth. Am J Dent 2000;13(1):28-34.

16. American Academy of Pediatric Dentistry. Clinical guidelines on pulp therapy for primary and permanent teeth: Reference manual 2006-07. Pediatr Dent 2006;28:144-148.

17. Geurtsen W, Spahl W, Leyhausen G. Residual monomer/additive release and variability in cytotoxicity of light-curing glass-ionomer cements and compomers. J Dent Res 1998;77(12):2012-2019. DOI: 10.1177/00220345980770121001.

18. Stanislawski L, Daniau X, Lauti A, et al. Factors responsible for pulp cell cytotoxicity induced by resin-modified glass ionomer cements. J Biomed Mater Res 1999;48(3):277-288. DOI: 10.1002/(sici)10974636(1999)48:33.0.co;2-t.

19. Shameem A, Muliyar S, Thankachan RP, et al. Study to evaluate the efficacy of resin-modified glass lonomer cement liner as a direct pulp capping material. J Contemp Dent Pract 2018;19(9):1065-1071. DOI: 10.5005/jp-journals-10024-2382.

20. Momoi Y, Hirosaki K, Kohno A, et al. Flexural properties of resinmodified "hybrid" glass-ionomers in comparison with conventional acid-base glass-ionomers. Dent Mater J 1995;14(2):109-119. DOI: 10.4012/dmj.14.109.

21. Yasuda $Y$, Ogawa M, Arakawa T, et al. The effect of mineral trioxide aggregate on the mineralization ability of rat dental pulp cells: an in vitro study. J Endod 2008;34(9):1057-1060. DOI: 10.1016/j. joen.2008.06.007.

22. Torabinejad $M$, Hong CU, McDonald F, et al. Physical and chemical properties of a new root-end filling material. J Endod 1995;21(7):349353. DOI: 10.1016/S0099-2399(06)80967-2.

23. Parirokh M, Torabinejad $M$. Mineral trioxide aggregate: a comprehensive literature review--Part I: chemical, physical, and antibacterial properties. J Endod 2010;36(1):16-27. DOI: 10.1016/j. joen.2009.09.006.

24. Cox CF, Sübay RK, Ostro E, et al. Tunnel defects in dentin bridges: their formation following direct pulp capping. Oper Dent 1996;21(1):4-11.

25. Mohammadi Z, Shalavi S. Antifungal effects of root canal irrigants and medicaments. An update review. N Y State Dent J 2014;80(5):58-63. 